\title{
PRÁCTICA PEDAGÓGICA DE LA EDUCACIÓN ECUATORIANA EN EL SIGLO XXI
}

\section{PEDAGOGICAL PRACTICE OF ECUADORIAN EDUCATION IN THE 21ST CENTURY}

\begin{abstract}
ב"
Derling José Mendoza Velazco ${ }^{a^{*}}$, Elizeth Mayrene Flores Hinostroza ${ }^{b l}$, Lorena Soledad Revilla ${ }^{b 2}$, Magda Francisca Cejas Martínez ${ }^{c}$ y Mercedes Navarro Cejas

Fechas de recepción y aceptación: 16 de noviembre de 2019, 23 de abril de 2020

DOI: 10.46583/edetania_2020.57.437

Resumen: El objetivo del estudio es brindar una aproximación teórica al modelo pedagógico aplicado de la educación básica ecuatoriana en el transcurso del siglo XXI. La investigación fue de tipo cualitativo, resguardado en la metodología fenomenológica. Los datos obtenidos fueron proporcionados por diez informantes clave, seleccionados de forma no probabilística intencional, como docentes activos egresados de distintas universidades tanto fiscales como particulares. Como instrumento clave para la recolección de datos se utilizó una entrevista de tipo semiestructurado. Los resultados fueron analizados de manera interpretativa por los investigadores en conjunto con el sistema operativo ATLAS.ti, los cuales permitieron la categorización de los resultados para estructurar una aproximación teórica de las deducciones informativas de la investigación.
\end{abstract}

${ }^{a}$ Instituto de Investigaciones Científicas y Tecnológicas. Universidad Nacional de Educación Ecuador (UNAE). Orcid: https://orcid.org/0000-0001-8275-3687.

${ }^{*}$ Correspondencia: Parroquia Javier Loyola (sector Chuquipata) Azogues. Cañar. Ecuador.

E-mail: derling969@gmail.com

${ }^{\mathrm{b}}$ Facultad de Educación Básica. Universidad Nacional de Educación Ecuador (UNAE).

${ }^{1}$ Orcid: https://orcid.org/0000-0003-2171-8348. ${ }^{2}$ Orcid: https://orcid.org/0000-0001-9197-2259.

${ }^{c}$ Facultad de Ciencias Empresariales. Universidad Nacional de Chimborazo. Orcid: https://orcid. org/0000-0002-0618-3608

${ }^{\text {d }}$ Facultad de Jurisprudencia. Universidad Regional Autónoma de los Andes (UNIANDES). Orcid: https://orcid.org/0000-0003-4377-7250 
Palabras clave: pedagogía, modelo educativo, paradigmas de la investigación, currículo, Ecuador.

Abstract: The objective of the study is to provide a theoretical approach to the applied pedagogical model of Ecuadorian basic education in the course of the 21 st century. The research was of a qualitative type, based on phenomenological methodology. The data obtained were provided by 10 key informants, selected in a non-probabilistic manner, as active teachers who had graduated from different universities, both public and private. A semi-structured interview was used as a key instrument for data collection. The results were analyzed in an interpretative way by the researchers in conjunction with the ATLAS.ti operating system, which allowed the categorization of the results to structure a theoretical approximation of the informative deductions of the research.

Keywords: Pedagogy, educational model, paradigms of research, curriculum, Ecuador.

\section{INTRODUCCIÓN}

La sociedad actual representa la amalgama de múltiples formas de pensamiento y dicciones culturales tan diversas como los grupos humanos que la constituyen. En este sentido, la integración del hombre a las nuevas exigencias sociales del siglo XXI le implica que asuma de manera crítica y consciente las transformaciones que suceden en su entorno social, lo cual enlaza la necesidad de resignificar los modelos y esquemas de pensamiento, pues emergen nuevos códigos de interpretación de las relaciones sociales, y simultáneamente se descalifican los que están vigentes (Caballero y Fernández, 2018).

Existe una dualidad, donde el sujeto tiende a satisfacer una necesidad de apropiación de nuevos elementos para el entendimiento, y a la vez busca su reacomodo cognitivo tanto en el conocimiento como en el uso y apoderamiento de referentes en los recientes campos profesionales, así como una formación específica y necesaria para que asuma una labor profesional con eficiencia y calidad, en justa proporción con los intereses individuales y colectivos del contexto de actuación.

Lo anterior alienta la importancia de asumir transformaciones de acuerdo con los cambios que se suscitan en Ecuador, pues se viven momentos de profundos cambios, orientados a la consolidación de una sociedad humanista, democrática, protagónica, participativa, multiétnica, pluricultural, plurilingüe e intercultural, en una república de derecho y de justicia, donde la educa- 
ción orienta la formación de ciudadanos con principios, virtudes y valores de libertad, cooperación, solidaridad, convivencia, unidad e integración, que garanticen la dignidad, el bienestar individual y colectivo; principios estos sustentados en el preámbulo de la Constitución de la República del Ecuador (2008), como modelo de sociedad que procura la consolidación de un desarrollo social y cultural sostenible.

La educación ante estos escenarios de transformación, pretende una permanente construcción en la que niños, niñas, adolescentes y adultos son asumidos en su integralidad y complejidad, espacio en el cual se consideran las experiencias educativas que conllevan al desarrollo de conocimientos, valores, actitudes, virtudes, habilidades y destrezas; la labor docente toma un papel protagónico, orientada al desarrollo de una práctica pedagógica, cónsona con los requerimientos sociales y culturales, que permita la formación de estudiantes, con la participación de todos los actores vinculantes con el proceso educativo, siendo estos padres, representantes, personas actuantes y significantes de la comunidad en pro de la educación de los infantes (Londoño y Vélez, 2018).

La práctica pedagógica, en concordancia con las trasformaciones existentes, potencia el deber ser de la formación, la libre actuación, la discusión, el diálogo y la reflexión, en razón de la pluralidad de saberes, consolida hombres y mujeres aptos para vivir en sociedad; pues la práctica pedagógica es producto de una cultura, de una historia y ante ellas ontológicamente se presenta como una entidad compleja, dinámica, susceptible de cambios, con reestructuraciones como producto de la acción social y de la misma cultura de la cual forma parte.

Esos aspectos de la práctica pedagógica (Díaz, 2004a) se indican como la actividad cotidiana realizada por los docentes, orientada por un currículo, en un contexto escolar y social, dirigida a la construcción de saberes y formación de los estudiantes, como vía para el desarrollo personal y la convivencia social, lo cual evidencia una práctica pedagógica asociada a los contextos tanto escolar como social, tendiente a que se potencie el proceso formativo, y la vinculación docente con la comunidad pedagógica, ajustados a la concreción de las actividades cotidianas de los maestros, en justa reflexión con la construcción y reconstrucción de ese quehacer educativo vinculante. Para Díaz (2006): 
La práctica pedagógica se lleva a cabo todos los días y tiene como propósito fundamental la formación de los estudiantes en la medida en que se utilizan adecuadamente estrategias y metodologías que guían hacia el saber, bajo la orientación de un currículo bien estructurado (p. 248).

De ahí la importancia de una práctica pedagógica que esté en concordancia con el modelo de sociedad actual, que responda a los esquemas y orientaciones epistemológicas que dan formación a los ciudadanos con una fuerte y sólida identidad ecuatoriana, con una conciencia ciudadana de soberanía que reconoce sus derechos y responsabilidades, incluyendo la educación, con base en el artículo 10 del Reglamento General de la Ley Orgánica de Educación Intercultural ecuatoriana (2012), que indica que "Los currículos nacionales pueden complementarse de acuerdo con las especificidades culturales y peculiaridades propias de las diversas instituciones educativas que son parte del Sistema Nacional de Educación, en función de las particularidades del territorio en el que operan".

Con base en lo expuesto, podría considerarse que la práctica pedagógica es el conjunto de actividades que se realicen día tras día por parte del maestro, en la cual se conjugan habilidades, valores y conocimientos concretizados en estrategias didácticas. Para Gómez (2002, p. 7), "implica el encuentro y desencuentro cotidiano entre el maestro y el alumno, entre el ideal y la realidad, entre el presente y el futuro; es el espacio cotidiano de negociación de conocimientos, valores, y normas".

Para ello, el docente en su práctica cotidiana tiene presentes conocimientos que ha adquirido social y culturalmente, los cuales van más allá de su formación como maestro, de sus conocimientos técnicos, conocimientos más sutiles que entran en una interacción permanente entre lo afectivo, lo social y lo intelectual.

La práctica pedagógica orienta el desarrollo profesional docente, desde la transformación de la propia práctica, la producción de un conocimiento válido que se fundamente en los saberes científicos, culturales y educativos; procesos individuales y colectivos de reconstrucción racional del pensamiento y la teoría; actuación racional de las nuevas generaciones; valoración de nuevos enfoques y modelos pedagógicos, dándole un nuevo significado colectiva- 
mente a la realidad que no satisface, así como el desarrollo social e individual (Zambrano, 2018).

En la actualidad, el docente desarrolla su actividad de formación en un contexto social en el que confluyen los conflictos, las preferencias culturales y políticas, las agrupaciones diversas, las creencias, las ideologías y las instituciones, por lo cual cada vez que se habla sobre la educación es frecuente escuchar lo que debe hacerse, pero las propuestas de cómo hacerlo son escasas, situación que activa la idea de los problemas en educación de forma cíclica. De ahí la importancia de la presente investigación, que tiene como objetivo brindar una aproximación teórica del modelo pedagógico aplicado en la educación básica ecuatoriana en el transcurso del siglo XXI.

\section{Sustento TEÓRICO DE LA INVESTIGACiÓN}

\subsection{Visión paradigmática de la investigación}

En la investigación educativa se plantea una visión paradigmática a través de dimensiones relacionadas con el objeto de conocimiento o la realidad que se desea estudiar. En este sentido, la visión paradigmática de esta investigación se planteó desde las dimensiones ontológica, epistemológica, teórica, metodológica y axiológica; en razón de un objeto de estudio representado por la práctica pedagógica, sujeto docente, educación básica.

\subsection{Dimensión ontológica}

Ontológicamente, se especifica la forma y la naturaleza de la realidad social y natural preguntándose cómo es ontológicamente la práctica pedagógica. Fueron los griegos quienes hicieron los aportes que dan sentido histórico a la práctica pedagógica; ellos centraron la práctica en el desarrollo de la politiké, es decir, la política entendida como toda acción que se está implementando, luego hicieron la propuesta de la tejné, ilustrada como la disposición interna y externa que guía la acción. 
Villar (2006, p. 201) expresa que "la madurez del pensamiento griego en cuanto a su comprensión de la práctica, llegó a proponer la praxis como toda acción que se crea para reconstruir las condiciones sociales del ser humano". Es decir, la praxis tiene el compromiso de rehacer y reinventar la acción; es una disposición ética frente a las acciones concretas caracterizada por la relación dialéctica entre el pensamiento y la acción, cuyo propósito es la reconstrucción permanente de los procesos históricos que se dan en situaciones sociales reales.

Considerando que la práctica pedagógica es producto de una cultura y de una historia, y ante ella se presenta ontológicamente como una entidad compleja, dinámica, susceptible de cambios y de reestructuraciones como producto de la acción social y de la propia cultura de la cual forma parte. Asimismo, es operante profundizar en determinados interrogantes: ¿cómo se origina la práctica pedagógica en el interior del sujeto docente?, ¿cómo se desarrolla la práctica pedagógica?, ¿qué elementos la conforman?, ¿qué caracteriza la práctica pedagógica?, y su implicación en la formación del sujeto docente en el nivel de educación primaria.

En atención a lo expuesto, asumir la investigación que se pretende desde un nivel ontológico tiene implícito ahondar en el concepto de hombre y de educación que da sentido a la práctica pedagógica, pues el objeto de la educación es el hombre, concebido como una unidad biópsico-espiritual, abierto a la trascendencia, es decir, entendido como persona, y como tal un ser único, irrepetible, sujeto de derechos y deberes inviolables, y que le da sentido a una construcción social desde sus actuaciones en la educación primaria.

\subsection{Dimensión epistemológica}

Epistemológicamente, el docente pedagogo siempre está en relación con el conocimiento práctico. ¿Cómo se realiza el abordaje y la problematización de la realidad? ¿Cuál es la lógica del conocimiento? Acercarse al razonamiento de la realidad como totalidad articulada y en movimiento, en el que la práctica pedagógica juega un papel primordial. De ahí que la investigación se sustente epistemológicamente en el paradigma sistémico, el paradigma constructivista y el paradigma humanista. 


\subsection{Paradigma sistémico}

En un sistema se da un conjunto de unidades interrelacionadas de modo que el comportamiento de cada parte depende del estado de todas las otras, pues todas se encuentran en una estructura que las interconectan según Bertalanffy (1981). Estos elementos, relacionados entre sí, constituyen una determinada formación integral no implícita en los componentes que la forman.

El modo como se abordan los objetos y fenómenos en el paradigma sistémico no puede ser aislado, sino que tiene que verse como parte de un todo. No es la suma de elementos sino un conjunto de ellos que se encuentran en interacción de manera integral, que producen nuevas cualidades con características diferentes, cuyo resultado es superior al de los componentes que lo forman y provocan un salto de calidad. Para Martínez (2006, p. 80) "lo sistémico se traduce en un nuevo paradigma científico, una teoría formal, y como tal implica una nueva forma de pensar, de mirar al mundo y metodología innovadora".

En este sentido, el proceso docente en su práctica educativa se organiza sobre bases científicas y con un carácter sistémico, que se establece con un criterio lógico y pedagógico, de tal manera que se pueda lograr la máxima efectividad en la construcción de los conocimientos y el desarrollo de habilidades.

\subsection{Paradigma constructivista}

El término constructivismo es reservado por Crotty (1998, p. 58) para "las consideraciones epistemológicas que se centran exclusivamente en la actividad de la mente individual para generar significado", y el término constituye una posición epistemológica, es decir, referente a cómo se origina y cómo se modifica el conocimiento.

Por tanto, el ser humano realiza constantes esfuerzos activos con la finalidad de interpretar la experiencia, buscando propósitos y significados a los acontecimientos que les rodean, y el conocimiento representa una relación directa del individuo con el mundo que experimenta. Entonces, a través del paradigma constructivista se provee una alternativa al propio concepto de 
conocimiento y de conocer en la que el conocimiento no es un objeto finito, sino una acción o un proceso de construcción situada y social.

El constructivismo como posición epistemológica se refiere al ser de las cosas, a cómo suceden. Santisteban (2017, p. 32) expone que "el constructivismo se plantea como el conocimiento se construye a través de la realidad, que se logra a través de la actuación sobre la misma, experimentando con situaciones y objetos, y al mismo tiempo transformándolos". De ahí que el papel del sujeto docente en la educación no es solo de transmisión de conocimientos; su función consiste en crear condiciones adecuadas para que el estudiante, en interacción con el propio docente, con apoyo en herramientas y material instruccional, entre otros elementos mediadores de saberes, alimente su construcción personal.

En concordancia con lo expresado, el paradigma constructivista refuerza la investigación en un intento de explicar cuál es la naturaleza del conocimiento humano, apoyado en la construcción de que una persona que aprende algo nuevo lo asocia con sus experiencias previas y a sus propias estructuras mentales, y esto lo puede lograr a través del lenguaje como herramienta cultural de aprendizaje donde el individuo construye su conocimiento porque es capaz de leer, escribir, preguntar a otros y preguntarse a sí mismo sobre aquellos asuntos que le interesan, pues literalmente se ha enseñado al sujeto a construir el conocimiento a través del diálogo continuo con otros seres vivos.

\subsection{Paradigma humanista}

La educación implica relación con el otro, involucrarse, ser responsable de la trascendencia no solo personal sino conjunta. Es construir la totalidad del ser humano a partir del contacto con los demás, es custodiar la presencia de la humanidad en cada uno, es interrelación, es comunicación humana, es diálogo. Este encuentro continuo implica tener presente al otro y dentro del ambiente educativo, el estudio del ser humano se realiza de una manera integral como una totalidad, en permanente cambio y constante desarrollo.

Aizpuru y Monserrat (2008, p. 35) expresan que "el paradigma humanista postula a la persona como eje central del modelo", el cual considera al estudiante como centro de la actividad pedagógica, como ente individual, único y 
diferente a los demás. Esta singularidad debe ser respetada y potenciada pero también considerada como condicionante de su aprendizaje, pues el estudiante siempre tiene la necesidad de crecer en conocimiento, de forma autodeterminada, capaz de resolver problemas, es un participante activo durante todo el proceso de aprendizaje.

El paradigma sostiene que la educación ayuda a desarrollar la individualidad, apoya que los estudiantes se reconozcan como personas únicas; y contribuye a que desarrollen sus potencialidades. Por otra parte, el docente es considerado por el paradigma humanista como un modelo a seguir por los estudiantes y debe tener presente que cada estudiante es diferente, sus características, sus contextos y sus estilos de aprendizaje son distintos y por ello percibe al profesor de diferente manera.

En consecuencia, en el paradigma humanista tanto el profesor como el estudiante se conciben como personas libres, electivas y responsables de sus acciones, se conducen según sus percepciones y responden al ambiente no de forma objetiva, sino según el modo en que lo perciben y comprenden. Estos tienen un encuentro personal a través de los escenarios de aprendizaje en una relación humana, y esta es la que constituye a la persona, la que permite avanzar como género humano.

En este sentido, la práctica pedagógica es humanista, es un encuentro humano con las individualidades de cada hombre y apunta al desarrollo de las potencialidades, dimensiones y competencias, articuladas al sentido histórico-social y a las estructuras sociopolíticas de la educación.

\subsection{Dimensión teórica}

Teóricamente, la investigación se apoya en la teoría del pensamiento complejo (Morín, 1998) y se concibe como una racionalidad en el abordaje del mundo y del ser humano, consistente en la concreción de las partes en el todo mediante el establecimiento de relaciones entre ellas, teniendo en cuenta sus diferencias. Morín (1998) sostiene que la interdisciplinariedad es la clave para que se entienda la complejidad del mundo. De igual forma, el estudio teóricamente se sustenta en la teoría del constructivismo social de Vygotsky. Desde esta perspectiva se consideran los eventos individuales, según Acosta 
(2006), desde una concepción social, es decir, suponiendo que todos los actos humanos son sociales y que reflejan la cultura y la historia de un grupo.

\subsection{Dimensión axiológica}

Desde una dimensión axiológica, se considera que los valores morales son los que orientan el comportamiento humano, y guardan relación con el ejercicio de la reflexión crítica en torno a la realidad educativa y social. En el contexto de esta investigación se asume que a través del proceso de mediación pedagógica se favorece una participación dialógica entre docentes y estudiantes, propiciando los aportes de todas las voces en relación con los asuntos que se estén tratando.

Pero la educación tiene que ir más allá de encarar el desarrollo del aspecto intelectual, puesto que debe enfrentar el problema de una cultura en expansión, la cual es solidaria, es un modo de abrazar al otro, y en ese mismo abrazo reconocerse uno mismo, todo ello, según Camp (2004, p. 45), “no puede, ni debe transcurrir al margen de la dimensión ética, que es la primera prioridad de la cultura humana universal".

La cultura en general y por ende la escolar, como un modo de vida integrado se fundamenta básicamente en un conjunto de normas ideales de comportamientos representados por valores, los cuales expresan lo que el grupo realmente es, y de lo quiere y debe ser dentro de la práctica pedagógica.

\section{Metodología}

La investigación se desarrolló bajo un enfoque cualitativo, a través del método fenomenológico y apoyado por un estudio de campo. Para Hernández, Fernández y Baptista (2014, p. 358), "El enfoque cualitativo se selecciona cuando el propósito es examinar la forma en que los individuos perciben y experimentan los fenómenos que los rodean, profundizando en sus puntos de vista, interpretaciones y significados". Desde esta visión, se justifica el enfoque cualitativo en la presente investigación, pues permitió a los investigadores centrar su interés en el entorno natural donde ocurren los hechos con 
la finalidad de encontrar una descripción relevante y propia de las situaciones que acontecen durante el desarrollo de la práctica pedagógica.

En cuanto al método fenomenológico, este permitió hacer énfasis en los aspectos individuales y subjetivos de la experiencia, es decir, cómo son las prácticas pedagógicas de los docentes. Permite el estudio de los fenómenos tal como son experimentados, vividos y percibidos por los sujetos, describe el significado de las experiencias vividas por una persona o grupos de personas, en este caso, los docentes, acerca de un concepto o fenómeno; es decir, se toma la fenomenología de la vida cotidiana pues cada hecho social solo es comprensible en su contexto, existe una comprensión del sentido común o significado del mundo de la vida cotidiana.

En cuanto a la investigación de campo, la Universidad Pedagógica Experimental Libertador (UPEL, 2010, p. 16) la define como:

El análisis sistemático de problemas de la realidad, con el propósito bien sea de describirlos, interpretarlos, entender su naturaleza y factores constituyentes, explicar sus causas y efectos, o predecir su ocurrencia, haciendo uso de métodos característicos de cualquiera de los paradigmas o enfoques de investigación conocidos.

De esta forma, los datos o información fueron recogidos directamente de la realidad investigada, la cual está representada en esta investigación por la realidad educativa donde los docentes de educación construyen sus prácticas pedagógicas.

\subsection{Sujetos de la investigación}

Para el diagnóstico, que fue producto del trabajo de campo, se escogieron los sujetos informantes a través de una muestra cualitativa, la cual, según Hernández, Fernández y Baptista (2010, p. 384), consiste en "grupo de personas, eventos, sucesos, comunidades, etc., sobre el cual se habrán de recolectar los datos, sin que necesariamente sea estadísticamente representativo del universo o población que se estudia".

Al respecto, se seleccionaron diez sujetos informantes, con la finalidad de recabar información para indagar cómo son las concepciones, vivencias y 
experiencias en relación con la práctica pedagógica del sujeto docente, en el nivel de educación general básica. Para ello se tomaron en cuenta los factores propuestos por Hernández, Fernández y Baptista (2014), que intervienen para la selección de los sujetos de investigación: 1) capacidad operativa de recolección y análisis; 2) entendimiento del fenómeno, y 3) naturaleza del fenómeno bajo análisis.

Esta muestra comprehensiva también tuvo criterios como que algunos sujetos informantes son sujetos docentes que se desempeñan en el nivel de educación básica, otros con gran experiencia en este nivel pero que actualmente trabajan como docentes universitarios en escuela de educación, en contextos variados desde rural, urbano y universitario, con posgrados en diferentes áreas educativas, género tanto masculino como femenino.

\subsection{Técnicas e instrumentos}

Los investigadores aplicaron una entrevista de tipo semiestructurado a los informantes (tabla 1) con el objetivo de obtener datos ineludibles que permitan dar respuesta al objetivo de la investigación. Para Schubert y Röhl (2019) este tipo de entrevista - la semiestructurada- se caracteriza por ser simple, comprensible y abierta, sin depender de las categorizaciones preestablecidas.

Basado en este enfoque, los informantes respondieron a las preguntas de los investigadores sin que el proceso de entrevista influyera indebidamente en ellas (tabla 1). Las entrevistas fueron grabadas en formato MPEG-1 Audio Layer III (MP3). Para la transcripción de las grabaciones, los investigadores usaron el sistema Dragon Naturally Speaking versión 13. Los textos fueron codificados para cumplir con las normas éticas de la investigación Publishing Ethics Resource Kit (2020) de Elsevier, para evitar revelar información personal, método recomendado por Ziskin (2019) y Cejas, Mendoza, Navarro, Rogel y Ortega (2019), en estudios cualitativos.

Consecuentemente, se procedió a un análisis completo de los manifiestos obtenidos a través del contraste o triangulación de los textos. Se hizo una distinción entre los textos obtenidos por los docentes informantes y los enfoques interpretativos adoptados por los investigadores. De esta manera emergió una aproximación teórica que respondió a lo establecido en el objetivo del estu- 
dio propuesto. Este método de contrastación fue recomendado por Schmidt y Wiesse (2019).

TABLA 1

Preguntas de la entrevista aplicadas a los informantes de la investigación

\begin{tabular}{|l|l|}
\hline \multicolumn{2}{|l|}{ Investigación: Práctica pedagógica de la educación ecuatoriana en el siglo XXI } \\
\hline Universidad Nacional de Educación (UNAE) & Fecha: \\
\hline Informante: & Lugar: \\
\hline Pregunta: & \\
\hline 1. ¿Cómo define las prácticas pedagógicas del ecuador? & \\
\hline 2. ¿Qué tipo de práctica pedagógica se desarrolla en su institución educativa? \\
\hline $\begin{array}{l}\text { 3. ¿Al concluir las practicas pedagógicas, se ofrecen espacios para la reflexión y discusión de las } \\
\text { problemáticas percibidas? }\end{array}$ \\
\hline
\end{tabular}

Fuente: Elaboración propia (2019).

\section{ANÁlisis de LOS RESUltados}

El conjunto de datos proporcionado por los docentes informantes a través de las entrevistas en profundidad que se realizaron en relación con sus prácticas pedagógicas, fueron examinados sistemáticamente para determinar partes y descubrir las relaciones entre estas y las relaciones con el todo, con lo cual se persigue un mayor conocimiento de la realidad educativa estudiada, así como avanzar mediante su comprensión hacia la interpretación de una aproximación teórica de la práctica pedagógica ecuatoriana en el siglo XXI.

Los autores de la presente investigación desarrollaron, como analistas, una serie de operaciones que no fueron definidas por un proceso lineal, sino que se dieron de manera simultánea, esto significa que el proceso que se propuso para tal fin solo tiene un carácter teórico. El proceso de análisis de los datos involucró la reducción de los datos, la organización y presentación y, por último, la interpretación y verificación. Para ello se siguió el procedimiento en tres fases planteado por la teoría fundamentada: codificación abierta, codificación axial y codificación selectiva. 
La fase de codificación abierta, según Strauss y Corbin (2002, p. 110), "es un proceso analítico por medio del cual se identifican los conceptos y se descubren en los datos sus propiedades y dimensiones". Es un proceso de involucramiento con la realidad objeto de estudio, donde comienza el proceso de construcción de categorías.

El proceso de categorización implica clasificar las partes en relación con el todo, describir categorías o clases significativas, diseñar y rediseñar, integrando y reintegrando el todo y las partes a medida que se lee el material, y de esta manera va emergiendo cada sector, evento, hecho o dato. De ahí que el trabajo estuvo centrado en un procedimiento de categorización y análisis de datos cualitativo ejercido por el software ATLAS.ti, versión 7. El programa facilitó la representación de redes estructuradas mediante la triangulación de los datos obtenidos para iniciar una previa codificación, la cual se fue depurando en función de seleccionar aquellos datos pertinentes al objeto de estudio.

Los textos guardados en esa unidad fueron divididos en porciones o unidades temáticas, las cuales expresan una idea o un concepto para ser posteriormente codificado. Allí se inició el proceso de categorización codificando las citas, las cuales son identificadas con la letra D, identificando al docente con números de forma secuencial, mediante términos o expresiones breves, como una primera categoría descriptiva. Por ejemplo, el primer docente entrevistado se codificó como D1, el segundo entrevistado como D2 y así hasta el último docente, D10.

Para la estructuración de los datos y su interpretación los investigadores utilizaron los datos obtenidos de la realidad. Para ello se hizo necesario organizar y esquematizar las dimensiones en diagramas de flujo o redes estructurales para iniciar el análisis, atendiendo al análisis descriptivo o descripción normal, apoyado en el programa ATLAS.ti., donde se conservó la originalidad de la información. Luego se procedió a la contratación de los datos, donde se relacionaron los resultados del análisis descriptivo de cada unidad de análisis con las demás, así como con los resultados de los autores consultados en los contextos teóricos.

Por último, el proceso de aproximación teórica facilitó un todo coherente y lógico de los resultados de la investigación, la cual se mejoró con el aporte de autores reseñados en el marco teórico. Allí se aplicó la hermenéutica dialéctica, desde la realidad expresada por los autores, en contraste con lo expuesto 
por los investigadores y lo investigado por otros autores, así como los postulados teóricos que fundamentaron la investigación.

\subsection{Resultados}

En pro del entendimiento de la información que se expresa en la tabla 2, se detallan las diversas dimensiones, conformadas por códigos informativos textuales, a fin de dar sentido interpretativo y comprensivo a la fundamentación de subcategorías y categorías que emergen de la indagatoria realizada.

En este sentido, la apertura de cada dimensión para el análisis se detalló de la siguiente manera:

- Un gráfico de información donde se expresan los códigos que conforman dicha categoría.

- Descripción del código detallado.

- Transcripción textual de información que identifica el código.

- Comentario global producto del análisis realizado a la información que conforma el código informativo.

TABLA 2

Codificación de la información

\begin{tabular}{|c|c|c|c|}
\hline & Dimensiones & Subcategorias & Categorias \\
\hline 1 & Práctica rutinaria & \multirow{4}{*}{ Tradicional } & \multirow{11}{*}{$\begin{array}{l}\text { Modalidades } \\
\text { de la práctica }\end{array}$} \\
\hline 2 & Práctica descontextualizada & & \\
\hline 3 & Práctica poco reflexiva & & \\
\hline 4 & Práctica tradicional & & \\
\hline 5 & Práctica como experiencia & \multirow{7}{*}{ Contextual } & \\
\hline 6 & Práctica contextualizada & & \\
\hline 7 & Práctica en contextos rurales & & \\
\hline 8 & Práctica flexible & & \\
\hline 9 & Práctica humanizada & & \\
\hline 10 & Práctica multirreferencial & & \\
\hline 11 & Vivencia docente & & \\
\hline
\end{tabular}




\begin{tabular}{|c|c|c|c|}
\hline & Dimensiones & Subcategorias & Categorias \\
\hline 12 & Actitud favorable hacia la reflexión & \multirow{2}{*}{ Apertura al cambio } & \multirow{12}{*}{$\begin{array}{l}\text { Reflexión } \\
\text { sobre la práctica }\end{array}$} \\
\hline 13 & Disposición abierta a cambios & & \\
\hline 14 & Condiciones de la reflexión & \multirow{5}{*}{ Práctica deliberativa } & \\
\hline 15 & Investigar para reflexionar & & \\
\hline 16 & Práctica reflexiva & & \\
\hline 17 & Reflexión sobre el hacer docente & & \\
\hline 18 & Reflexión para mejorar & & \\
\hline 19 & Escasa formación docente & \multirow{5}{*}{$\begin{array}{l}\text { Barreras } \\
\text { para la reflexión }\end{array}$} & \\
\hline 20 & Escasa reflexión & & \\
\hline 21 & Conocimiento docente limitado & & \\
\hline 22 & Poco tiempo para reflexionar & & \\
\hline 23 & Relación teoría-práctica & & \\
\hline 24 & Actores de la práctica & Agentes educativos & \multirow{4}{*}{ Currículo } \\
\hline 25 & Proyectos de aprendizaje & \multirow{3}{*}{ Enseñanza } & \\
\hline 26 & Práctica evaluativa & & \\
\hline 27 & Formación docente & & \\
\hline 28 & Alcances de la práctica pedagógica & \multirow{3}{*}{$\begin{array}{l}\text { Características } \\
\text { de la práctica }\end{array}$} & \multirow{7}{*}{$\begin{array}{l}\text { Concepciones } \\
\text { de la práctica }\end{array}$} \\
\hline 29 & Rasgos de la práctica pedagógica & & \\
\hline 30 & Elementos de la práctica pedagógica & & \\
\hline 31 & Concepciones sobre la práctica pedagógica & \multirow{4}{*}{$\begin{array}{l}\text { Creencias } \\
\text { sobre la práctica }\end{array}$} & \\
\hline 32 & Concepciones de la educación primaria & & \\
\hline 33 & Cuestionamiento del rol del docente & & \\
\hline 34 & Naturaleza de la práctica pedagógica & & \\
\hline
\end{tabular}

Fuente: Elaboración propia (2019).

\subsection{Categoría: modalidades de la práctica pedagógica}

Las subcategoría práctica pedagógica tradicional y práctica pedagógica contextualizada se agrupan en una categoría llamada Modalidades de la práctica pedagógica, cuya descripción se presenta en la figura 1 . 
FIGURA 1

Categoría: Modalidades de la práctica pedagógica

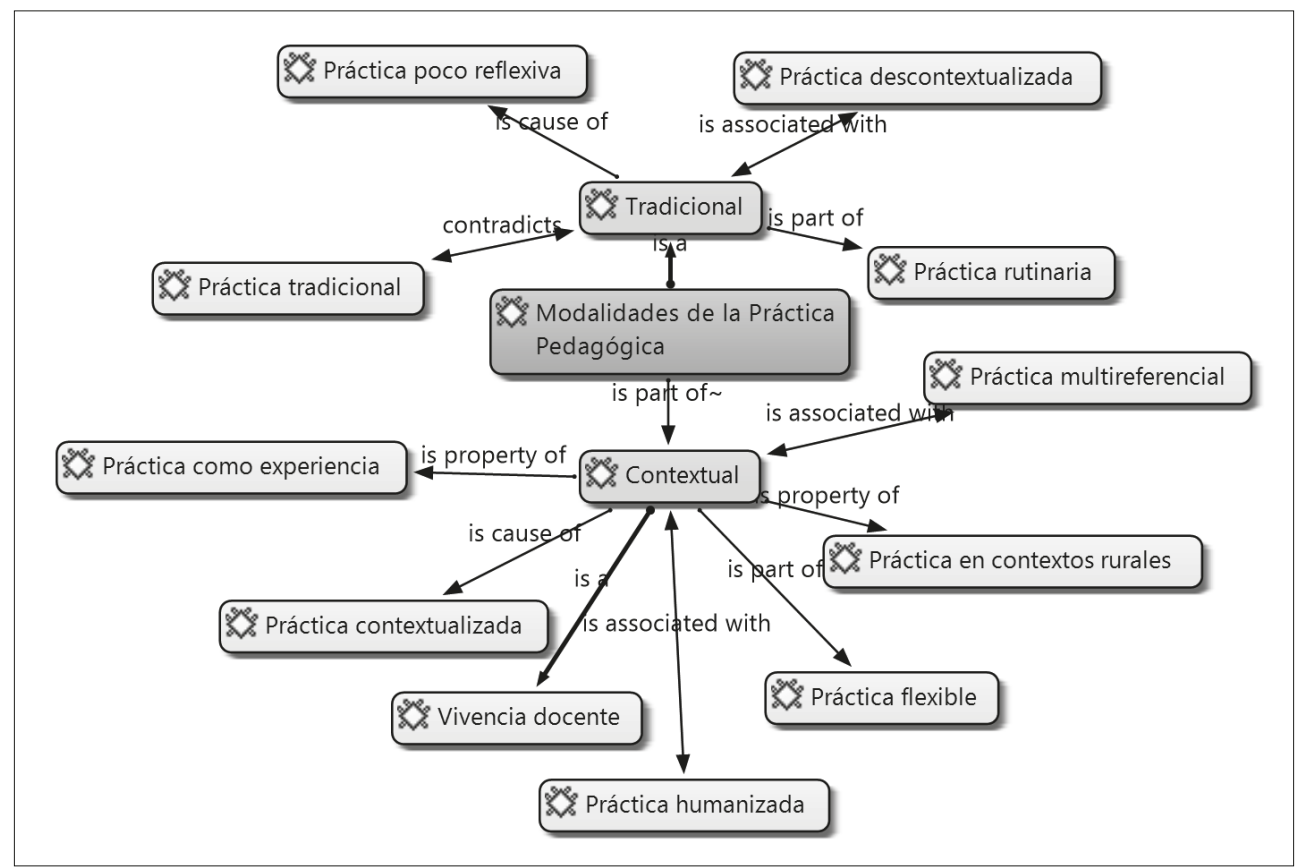

Fuente: Elaboración propia (2019).

En el quehacer diario que realizan los docentes interactúan diversos métodos, técnicas y estrategias para que se potencie la enseñanza y el aprendizaje en pos de una formación efectiva; se genera una práctica cotidiana multifacética y compleja donde convergen una variedad de acciones que llevan a la práctica pedagógica a ubicarse en una modalidad que muestra cómo los sujetos docentes asumen posturas diversas ante el proceso pedagógico.

Entonces, una modalidad, en palabras de Ávalos (2005: 23), "hace referencia a los campos que delimitan un modo particular de cómo ser, hacer, conocer y convivir". A efectos de denominación una modalidad de práctica pedagógica se asume como el modo particular de realizar las prácticas pedagógicas y contempla el tiempo, modo y espacio en el cual esta se realiza. La modalidad integra la tipología, caracterización, experimentaciones, construcciones 
teóricas, conceptuales, investigaciones, avances relacionados entre la pedagogía, el área de conocimiento específica y escenarios particulares.

Bajo esta concepción, las modalidades de la práctica pedagógica emergen desde los hechos que realizan los sujetos docentes. En esa diversidad de hechos, según los testimonios de los informantes, domina el tipo de formación docente recibida, las experiencias vividas, el nivel y modalidad educativa que se atiende, las prescripciones del diseño curricular, la organización escolar y las características propias del docente desde sus valores, ideologías y actitudes, en un contexto histórico cultural producto de las interacciones personales e institucionales.

Estos elementos ubican la práctica pedagógica en una modalidad que, al ser detallada y contrastada con la fundamentación teórica, se denomina práctica pedagógica tradicional o conservadora. Esta modalidad se caracteriza de acuerdo con Isaza y Gómez (2005, p. 32), por ser prácticas que:

corresponden a una jerarquía explícita, las relaciones de poder están bien definidas, aunque tanto el maestro como el alumno deben aprender su rol. Se identifica una práctica transmisiva, de orden regulador y discursivo, y solo importa el producto externo del alumno, el texto que crea, el problema que resuelve, y la medida en la que éstos se ajustan a los criterios establecidos. Con cierta frecuencia se aplican exámenes a los alumnos con la finalidad de homogeneizar la adquisición de los contenidos o en determinado caso, crear estrategias para su adquisición, por ejemplo, buscar algún tipo de apoyo. Prevalece la utilización de un solo libro de texto, idéntico para todos, que corresponde a la secuencia establecida en el documento de planificación, casi sin apoyo de otros materiales. En las prácticas pedagógicas tradicionales se privilegia la adquisición de aprendizajes conceptuales y lógico-matemáticos, quizá por ser más fáciles de evaluar a través de pruebas objetivas, es decir, a una práctica pedagógica conservadora, que automáticamente excluye, llegando al extremo de repetición de grado o a la deserción escolar.

Según lo expresado, esta modalidad de práctica pedagógica expresa una diversidad que debe reconocerse como secuencias de acciones recurrentes que canalizan procesos de formación en el nivel de la educación básica. Estas manifestaciones tienen su génesis en el modelo pedagógico que asuma el docente, y en su ejecución se devela un empirismo pedagógico en el que 
existe escasa reflexión del proceso, un seriado de contenidos que en ocasiones se convierten en enciclopedismo exagerado, para luego conectarlo con una memorización con poca trascendencia para el estudiante, razón que da valoración al verbalismo al saber hablar sobre los datos de la cultura, a diferencia de la utilización práctica de ese saber en situaciones reales de la vida y en contacto con su entorno, es decir, se presentan actividades planificadas.

Según los testimonios del docente informante D2, "las actividades tienen una planificación que en el cuaderno está bien, pero para nada la aplican. Eso es lamentable y ocurre en muchas instituciones educativas". Las prácticas pedagógicas son canalizadas a través de un modelo tradicional, las cuales se encuentran presentes en el docente, que guía su plano ideológico y actitudinal.

De igual forma para D7 “muchos profesores indagan tener prácticas constructivas, pero en realidad todos se basan en el manual de estudios, es como tener doble cara ante lo que hacemos en las aulas de clase". Estas actuaciones son recurrentes desde las primeras manifestaciones del arte de enseñar, y aunque las tendencias pedagógicas actuales sitúan desde el deber ser de actuación a un docente contrario a estas prácticas se siguen observando, en detrimento del proceso crean en los actores educativos diversas actuaciones poco efectivas desde lo pedagógico y didáctico, para que se encare una formación en niños y niñas del nivel de educación básica.

Se puede resaltar según los manifiestos, que es evidente la existencia de prácticas pedagógicas, caracterizadas por la repetición de acciones, la clase magistral como una vía de acceso al saber y la escasa utilización de recursos para apoyar la enseñanza; sigue imperando la idea de que todos los estudiantes aprenden de la misma manera, y no existe un diagnóstico apropiado que conecte el aprendizaje del estudiante con los fines educativos y con el nivel de maduración.

D10 señala: "yo he visto prácticas rutinarias, sin sentido, y poco efectivas, sí hablamos de formación para los niños". Por su parte, D9 asegura que "La repetición y la monotonía obstaculizan la práctica efectiva". Por lo tanto, es recurrente el uso de planificaciones educativas que solo cumplen una normativa de presentación, pero para nada trascienden en la formación y evaluaciones que solo dan cuenta de acciones netamente memorísticas, y mayor aún desde una gerencia de aula efectiva. Estas prácticas pedagógicas poco se 
conectan con la real educación básica necesaria en la formación del contexto que se indaga.

El informante D4 expone que "la práctica pedagógica se genera en muchas ocasiones a espaldas de lo que busca la institución a través del proyecto educativo, y alejada de los proyectos de aprendizaje". Esta recurrencia lleva al docente a prácticas pedagógicas que desde la indagatoria son caracterizadas como rutinarias, no están matizadas por la novedad, el ingenio, creatividad, y menos aún por la alternabilidad en la ejecución.

A lo expresado D8 añade que "las vivencias con la práctica pedagógica que se realizan, no tienen ninguna repercusión con la formación de los niños”, y para D5 "es en principio lo que orienta las prácticas, pues nos ocupamos solo de elementos secundarios como los mapas conceptuales, los deberes puntuales y perfectos". Esto trae consigo un impacto en la actitud de los estudiantes ante los procesos con dichas características, lo cual induce al cansancio, la escasa valoración de lo que se hace en el aula de clase. Las actividades, lejos de apoyar la formación, se convierten en fuertes responsabilidades contraídas por toda la familia en lo económico, por la solicitud y desarrollo de deberes que aglomeran altos costos.

La apatía, la actitud negativa, el irrespeto y la indisciplina son recurrentes en los estudiantes para D3, que expone que "los licenciados terminan haciendo otra cosa en el aula, o normalmente lo que producen es aburrimiento, rutina, y cansancio en los niños", y por ello las prácticas son contrarias al ideal, podrían convertirse en la génesis de un rendimiento escolar poco efectivo, y en caso extremo fuertes debilidades para que se alcance las competencias básicas necesarias, para que sean promovidos al nivel o grado inmediato superior.

De igual manera, se devela una modalidad contextual de la práctica pedagógica apoyada en el plano experiencial, donde el sujeto docente desde su plano ideológico, actitudinal y sus vivencias crea un ideario pedagógico de actuación que canaliza su quehacer de formación.

La práctica pedagógica contextualizada trata de dar respuesta a las exigencias del proceso de formación desde un orden lógico de actuación, con escasa fundamentación teórica, pero tratando que se entiendan los procesos, en asocio con la experiencia que le asiste a cada docente. En otras palabras, una validación del quehacer educativo desde la construcción que realiza el 
maestro, en pro de un estado ideal del proceso de actuación de formación. Situación que se devela desde las características de esta modalidad de práctica pedagógica, donde la práctica como experiencia cobra un significado mayor, pues se trata de ir en busca de un saber pedagógico que evidentemente es asistido por la experiencia, aunque esta es intrínseca en el sujeto y se asume como condición natural; debe ser bien canalizada, donde el plano de lo experiencial sea el soporte para que se canalicen los procesos y no una posible experiencia signada solo por los años de servicio, a espaldas de lo enriquecedor de la práctica, y mayor aún, lo que contribuye a la formación como docente.

Es así como lo indica D6: "una práctica pedagógica debe estar sujeta a constantes cambios, pues es allí donde esta parte de la experiencia, hay que ir incorporando las nuevas experiencias en lo que se hace". La práctica pedagógica toma características de proceso cíclico, donde lo que ocurre desde la experiencia docente debe ser valorado, sistematizado y convertirse en el punto de partida de un saber docente que, al contrastarse con la práctica, devela un conocimiento que ofrece teoría pedagógica, digna de ser asumida por otros sujetos, y diversos colectivos educativos.

\subsection{Categoría: reflexión sobre la práctica pedagógica}

Las subcategorías de apertura al cambio, práctica pedagógica deliberativa y barreras para la reflexión forman la categoría llamada reflexión sobre la práctica pedagógica, cuya descripción puede verse en la figura 2.

En el plano pedagógico, para Orriego (2007) la acción reflexiva se ha orientado al análisis teórico y práctico acerca de cómo se entiende y favorece el repensar en la actividad pedagógica. Para D5 "Una práctica pedagógica debe responder a la formación de los estudiantes en el contexto de la reflexión educativa", de esta forma se percibe el valor de lo reflexivo como cualidad pedagógica, y como método o recurso para el trabajo de los profesionales de la educación en la dirección del aprendizaje y en el desarrollo de la personalidad de los educandos. 
FIGURA 2

Categoría: reflexión sobre la práctica pedagógica

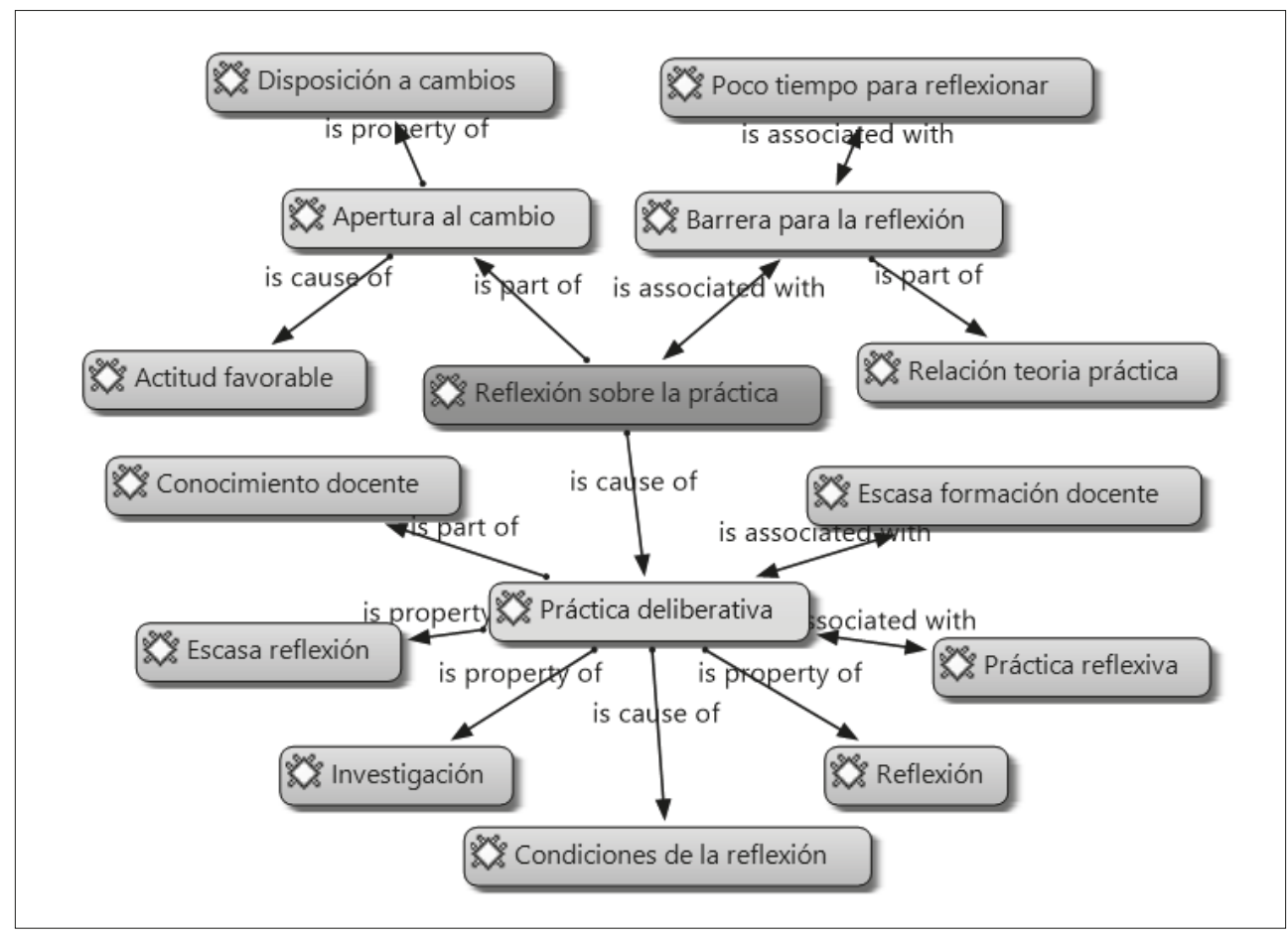

Fuente: Elaboración propia (2019).

En este sentido, D1 señala que "el docente tiene la oportunidad de reflexionar en los núcleos problemáticos y de las estrategias y recursos, para hacer que esa práctica pedagógica tenga sentido, y sea pertinente para la educación"; de esta manera el sujeto docente hace énfasis en su persona como sujeto reflexivo de lo que hace, de su actuación personal y pedagógica. La reflexión pedagógica emerge entonces como un proceso esencial de su propia formación y del otro, es decir, de los educadores y de su rol en la formación de los educandos.

Respecto a los espacios de reflexión, según D6 "En mi práctica pedagógica está una gran debilidad inicial, es la falta de tiempo para reflexionar con el estudiantado". La limitación del tiempo, según el docente informante, conlleva 
las subcategorías de práctica deliberativa y barrera para la reflexión, es decir, el factor de restricción en la formación se ciñe a recetas estandarizadas sobre cómo mediar proceso de enseñanza en menos tiempo, dejando a un lado las particularidades propias del contexto, en ocasiones en total desapego con la normativa y el currículo que orienta el nivel educativo ecuatoriano con más amplitud en la investigación.

La subcategoría de apertura al cambio se refleja cuando D7 expone que "si existe reflexión en las prácticas educativas existen cambios en la educación"; en resumen, la reflexión sobre lo cotidiano que realiza el docente en los espacios educativos genera cambios desde la base de los hechos que se transforman con reflexiones operativas que tienen su origen en la resignificación educativa, y que tienden a que se realice una práctica pedagógica cónsona con la plataforma curricular existente y el deber ser de educación donde la interdisciplinariedad orienta una vía expedita para el fortalecimiento de un encuentro crítico y fecundo de las distintas perspectivas y variantes que caracterizan el proceso de conocimiento asociado con la práctica pedagógica.

\subsection{Categoría: currículo}

Las subcategorías de enseñanza y agentes educativos forman la categoría llamada currículo, cuya descripción se realiza en la figura 3.

Desde la formación, el currículo es el foco intelectual y organizativo de los procesos educativos en las instituciones escolares y constituye el espacio donde se definen y debaten los fines, contenidos y procesos que dan forma a una determinada concepción de la educación. Desde sus orígenes, el término currículo ha sido utilizado para significar diversas formas e instrumentos a través de los cuales se intenta la regulación sistemática e intencionalmente el contenido y las formas de las actividades de enseñanza.

En este sentido, el currículo, para Sacristán (2002, p. 2), en "la acción es la última expresión de su valor, pues es en la práctica donde todo proyecto, toda idea, toda intención, se hace realidad de una forma u otra". Por tanto, el currículo, al expresarse a través de la práctica, genera un definitivo significado para los estudiantes y docentes desde la diversidad de acciones didácticas que se realizan en los espacios de formación. 
FIGURA 3

Categoría: currículo

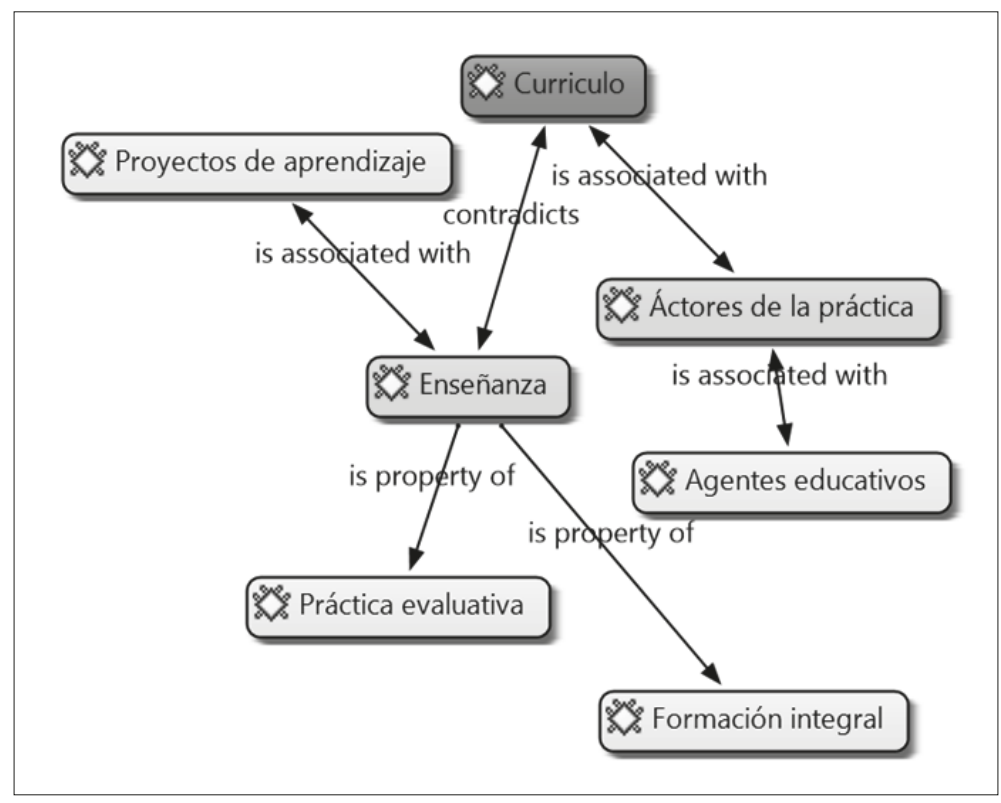

Fuente: Elaboración propia (2019).

En el sistema educativo ecuatoriano, la aplicación curricular deriva de documentos denominados currículo de los niveles de educación obligatoria (2016), de aplicación en la realidad escolar del país, orienta las bases históricas, pedagógicas, filosóficas, sociales, culturales, psicológicas, políticas, metodológicas, científicas y humanistas, con las cuales se implementará la formación de los niños, niñas, jóvenes, adultos y adultas de nuestro país, en los subsistemas que le competen al Ministerio de Educación.

Para D10, "el currículo y el sistema educativo actual, están en contradicción, nos piden ser constructivos, pero... ¿cómo puedo ser constructivista 40 estudiantes en el aula?". La subcategoría enseñanza está a la deriva de la categoría currículo, pero en teoría se solicita ejercer un modelo educativo ideal para los estándares de educación ecuatoriana, y, en realidad, los docentes sustentan otra, es decir, los modelos conductistas. 
En este sentido, desde un plano teórico la educación básica se relaciona según la Organización de Estados Iberoamericanos para la Educación la Ciencia y la Cultura (2005), con el proceso de enseñar a pensar, enseñar a aprender, enseñar a ser y estar. Esto implica el desarrollo de diversas estrategias que incluyen el fomento de la creatividad, el sentido de responsabilidad, el fomento de la independencia en la búsqueda del conocimiento, la incentivación de un acercamiento interdisciplinario hacia el conocimiento y la posibilidad del desarrollo de las aspiraciones individuales.

En el modelo curricular se encuentran los actores de la práctica, considerados como subcategoría emergente. Estos actores presentan inquietudes según D9 al manifestar que "las prácticas deben ser libres, pero con normas y orientaciones". Y D4 opina que "la práctica pedagógica no debe ser una camisa de fuerza, debe ser flexible, no debe ser una copia todos los días".

Lo anterior da cuenta de la intencionalidad curricular desde la formación de sujetos en el nivel de educación básica, de ahí la aplicación real de un sustento curricular desde elementos culturales (conocimientos, valores, hábitos, creencias) que conforman una propuesta educativa y social, en pro de la formación de ciudadanos que debe canalizarse desde una práctica pedagógica que represente una verdadera intencionalidad curricular, y no un seriado de actividades con escaso impacto en la educación de los infantes.

\subsection{Categoría: concepciones de la práctica pedagógica}

Las subcategorías características de la práctica y creencias sobre la práctica forman la categoría de concepciones de la práctica pedagógica, cuya descripción se realiza en la figura 4.

Los docentes de educación básica poseen concepciones complejas sobre el proceso de práctica pedagógica, en las que coexisten variados modelos pedagógicos, referentes de planeación y acciones, muchas veces contradictorias, que dificultan procesos de aprendizaje y enseñanza de tipo indagador y reflexivo. En este sentido D3 indica que "la práctica pedagógica se genera desde las percepciones y actitudes de los docentes, pues los planos ideológicos se reflejan en la práctica pedagógica". De ahí que los docentes asuman 
creencias sobre la práctica con posturas constructivistas desde el discurso, pero subyace el desarrollo de una práctica pedagógica centrada en el desarrollo de actividades o la adquisición de contenidos y actitudes desde la práctica.

FIGURA 4

Categoría: concepciones de la práctica pedagógica

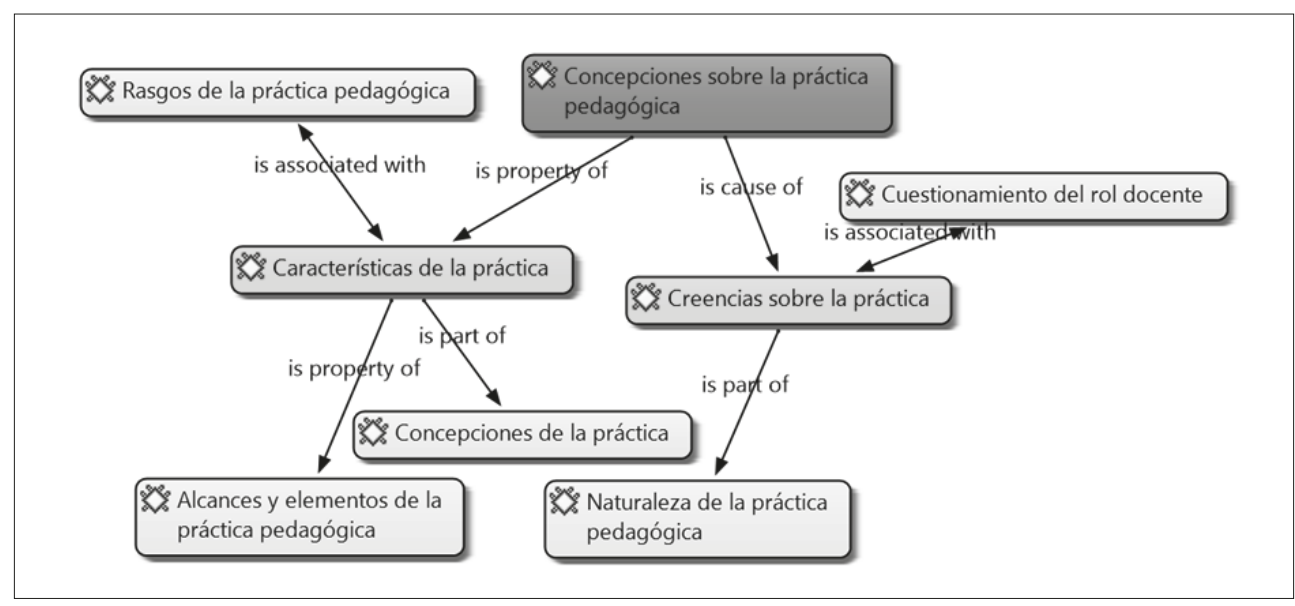

Fuente: Elaboración propia (2019).

Asimismo, desde el punto de vista de los modelos pedagógicos, la concepción que subyace con más fuerza es la instrumental, ya sea en la pedagogía tradicional o en la activa, que son las de mayor frecuencia, y en las que no se realiza una estructuración ideológica, intelectual ni pedagógica. En consecuencia, predomina la demostración de métodos según modelos de las corrientes que estén de moda: el conductismo, el cognitivismo o el constructivismo.

Estos modelos son conceptualizados por D7 al establecer que "la teoría induce a la práctica pedagógica, y esta debe tener unas características particulares que la definan". Los entrevistados definen la práctica pedagógica con características sumamente particulares que tienen su génesis en la concepción pedagógica que asuma el sujeto docente en justa correspondencia con su plano ideológico, desde las creencias que cultiva en su actuación de formación. Por lo tanto, las concepciones de la práctica pedagógica se expresan 
con creencias que se conectan con los lineamientos, normativas y diversas fuentes legales que sirven de punto de partida para la creación de un ideario de actuación que posibilita un impacto importante en los estudiantes. De ahí el seguimiento que se hace desde las concepciones docentes en razón de debilidades en las prácticas, el reconocimiento de ello, la posible reflexión de los procesos y los cuestionamientos en todo el colectivo docente que hacen vida profesional en las escuelas de educación básica.

\section{Aproximación teÓRICA de LAS CATEGORÍAS}

Las vivencias como fuente para encarar procesos pedagógicos toman valor protagónico en los resultados del estudio, pues permite un acercamiento a un saber para la formación; al respecto, para Díaz (2004b, p. 23), “expresa los conocimientos construidos de manera formal o informal por los docentes, fundamentado en sus valores, ideologías, actitudes, y prácticas en un contexto histórico cultural". Desde esta percepción, la práctica pedagógica se asiste del saber pedagógico, y este tiene su fuente en la experiencia, las vivencias y el conocimiento de los contextos de actuación, en justa correspondencia con la teoría pedagógica que permite reconocer cómo sucede la práctica y cómo darle un sentido verdaderamente de formación.

De lo expresado, se desprende la diversidad que emana de lo cotidiano de las prácticas pedagógicas en la educación básica ecuatoriana, lo cual requiere también que se diversifiquen esas acciones de formación partiendo del reconocimiento que los sujetos que aprenden son diferentes, aunque asistan a la misma escuela y estudien el mismo nivel escolar. La educación debe ser integradora de todos o, en caso contrario, traicionará el derecho universal de la educación. Por lo tanto, que se atienda la diversidad es un gran reto que implica grandes reconstrucciones de las prácticas pedagógicas, para que se logre una educación de calidad que respete las diferencias y evite la segregación.

Para los autores de la investigación, la realidad descrita desde la práctica pedagógica en el nivel de educación básica es poco reflexiva y se detalla como rutinaria, con vivencias poco impactantes, con una fuerte debilidad en los docentes desde la teoría pedagógica que manejan, lo cual le convierte en 
un sujeto con un ineficaz desempeño práctico y acciones complementarias tales como la estética literaria, carteleras bonitas, gran cantidad de deberes, actividades perfectas muchas veces no realizadas por los estudiantes, ni mucho menos orientadas debidamente por los docentes, ya que estos no poseen la autonomía epistémica para su diseño o corrección del material preimpuesto en la institución, donde pasa a ser lo importante en lo que se hace a diario en las escuelas, de hecho podría hablarse de un modelo de "escuela para actividades", es decir, todos los días múltiples actividades, pero poco impacto en la formación integral de los estudiantes de acuerdo con el nivel educativo.

Una práctica pedagógica de apertura, humanista y contextualizada, sin duda es la que responde adecuadamente a la diversidad, en conexión con el entramado curricular, y con la convicción de adaptarlo según el potencial de cada contexto y de cada estudiante. Bajo esta perspectiva de comprensión, la complejidad de la práctica pedagógica aplicada en la educación básica muestra evidentes aspectos que le caracterizan por tener una aproximación con un enfoque tradicional de mediación, donde la rutina, el enciclopedismo y las acciones de formación se encuentran poco contextualizadas.

En este sentido, para los investigadores las categorizaciones conllevan una aproximación teórica donde se resalta que es fundamental hacer una revisión del modo en que el docente concibe los procesos de enseñanza-aprendizaje, y la concepción que tiene de la práctica que hace a diario en la escuela, dada la orientación necesaria y continua de la práctica desde la acción dialéctica, a partir de la articulación de la practica con la fundamentación teórica, a fin que se devele el sentido ontológico de ese acto de formación. Para ello, es operativo que el sujeto docente, desde el deber ser de su compromiso profesional como mediador en pro del proceso de formación, induzca una reflexión sobre la práctica pedagógica que le permita conocer, interpretar, transformar y mejorar su currículo. Es decir, que se conceptualice y operacionalice un abordaje emancipador del continuo pedagógico, para el entendimiento y la resolución de los problemas relacionados con la práctica pedagógica, mediante la investigación, la reflexión crítica y toma de conciencia, orientada a la transformación diaria en las escuelas de educación básica ecuatoriana del siglo XXI. 


\section{BiBLIOGRAFÍA}

Acosta, M. (2006). El aprendizaje visto como un proceso de interacción social. Revista Ciencias de la Educación 6(1), Venezuela.

Aizpuru, C. y Monserrat, G. (2008). La persona como eje fundamental del paradigma humanista. Acta universitaria 18, 33-40.

Avalos, B. (2005). Renovando la formación docente inicial. Revista Colombiana de educación 47, 13-29.

BertalanfFy, Y. (1981). Tendencias en la teoría general de sistemas, Madrid: Alianza.

Caballero, P. y Fernández, M. (2018). Creatividad y rendimiento académico: un estudio de caso con alumnos de $4 .^{\circ}$ curso de educación secundaria. Revista Iberoamericana De Educación 78(2), 77-95.

CAMP, V. (2004). Los valores y la educación. México: Trillas.

Cejas, M., Mendoza, D., Navarro, M., Rogel, J. y Ortega, Y. (2019). A Performance-Centred Competency-Based Approach to Quality University Teaching. Integratsiya obrazovaniya 23(3), 350-365. DOI: <https://doi. org/10.15507/1991-9468.096.023.201903.350-365>.

Código de ética, PERK (2020). Kit de recursos de ética editorial para editores: Publishing Ethics Resource Kit (PERK) de Elsevier. Disponible en: $<$ https://www.elsevier.com/editors/perk $>$.

Constitución de la República del Ecuador (2008). Ciudad Alfaro, Asamblea Constituyente. Disponible en: $<$ https://www.oas.org/juridico/pdfs/mesicic4_ecu_const.pdf>.

Crotty, M. (1998). Los fundamentos de la investigación social: significado y perspectivas en el proceso de investigación. Londres: Sage.

Currículo de los niveles de educación obligatoria (2016). Ecuador: Ministerio de Educación.

DíAz, V. (2004a). Currículum, investigación y enseñanza en la formación docente. Venezuela: Universidad Pedagógica Experimental Libertador, UPEL.

Díaz, M. (2004b). Del discurso pedagógico: Problemas críticos. Bogotá: Colección Seminarium magisterio.

DíAz, V. (2006). Construcción del saber pedagógico, tesis doctoral, Venezuela: Universidad Pedagógica Experimental Libertador, UPEL. 
Gómez, E. (2002). La recuperación de la práctica educativa y la profesionalización de la actividad docente. Guadalajara: Centro de pedagogía Ignaciana del ITESO.

Hernández, R., Fernández, C. y Baptista. P. (2010). Metodología de la investigación. México: McGraw Hill.

Hernández, R., Fernández, C. y Baptista. P. (2014). Metodología de la investigación. México: McGraw Hill.

Isaza, L. y Gómez C. (2005). Práctica pedagógica: horizonte intelectual y espacio cultural. Medellín: Universidad de Antioquia.

Londoño, A. y Vélez, O. (2018). From the disjunction between passive and active instruction approaches towards their integration as an alternative for the significant learning of sustainable development. Culture and Education 30(4), 766-786. DOI: <https://doi.org/10.1080/11356405.2018.1514803>.

Martínez, M. (2006). Ciencia y arte de la metodología cualitativa. México: Trillas.

Morín, E. (1998). Introducción al pensamiento complejo. Barcelona: Gedisa Editorial.

Organización de Estados Iberoamericanos Para la Educación, la Ciencia y la Cultura (OEI) (2005).

ORRIEGO, J. (2007). La pedagogía como reflexión del ser en la educación latinoamericana. Manizales: Editorial Solar.

Reglamento general de la ley orgánica de educación intercultural ecuatoriana (2012). Ecuador: Ministerio de Educación.

SACRISTÁn, J. (2002). El Curriculum: una reflexión sobre la práctica. Madrid: Ediciones Morata.

SAntisteban, C. (2017). El socio-constructivismo y las prácticas didácticas en el curso de diseño arquitectónico. Revista AVANCE 11(2), 26-47.

Schubert C. y RöHL T. (2019). Ethnography and Organisations: Materiality and Change as Methodological Challenges. Qualitative Research 19(2), 164-181. DOI: <https://doi.org/10.1177/1468794117744748>.

Schmidt, R. y Wiesse, B. (2019). Online Participant Videos: A New Type of Data for Interpretative Social Research? Qualitative Social Research 20(2). DOI: <http://dx.doi.org/10.17169/fqs-20.2.3187>.

Strauss, A. y Corbin, J. (2002). Bases de la investigación. Colombia: Universidad de Antioquia. 
Universidad Pedagógica Experimental Libertador (2010). Manual de trabajos de grado, especialización, maestrías y tesis doctorales. Caracas: FedUPEL.

Villar, A. (2006). Ciencia y creencia. Madrid: Ediciones Pedagógicas.

Zambrano, E. (2018). Prácticas pedagógicas para el desarrollo de competencias ciudadanas. Revista Electrónica de Investigación Educativa 20(1), 69-82. DOI: <https://doi.org/10.24320/redie.2018.20.1.1409>.

ZIsKIN, M. (2019). Critical discourse analysis and critical qualitative inquiry: data analysis strategies for enhanced understanding of inference and meaning. International Journal of Qualitative Studies in Education 32(6): 606631. DOI: <https://doi.org/10.1080/09518398.2019.1609118>. 
